\title{
A frequency analysis of cone characteristics for the different stages of keratoconus
}

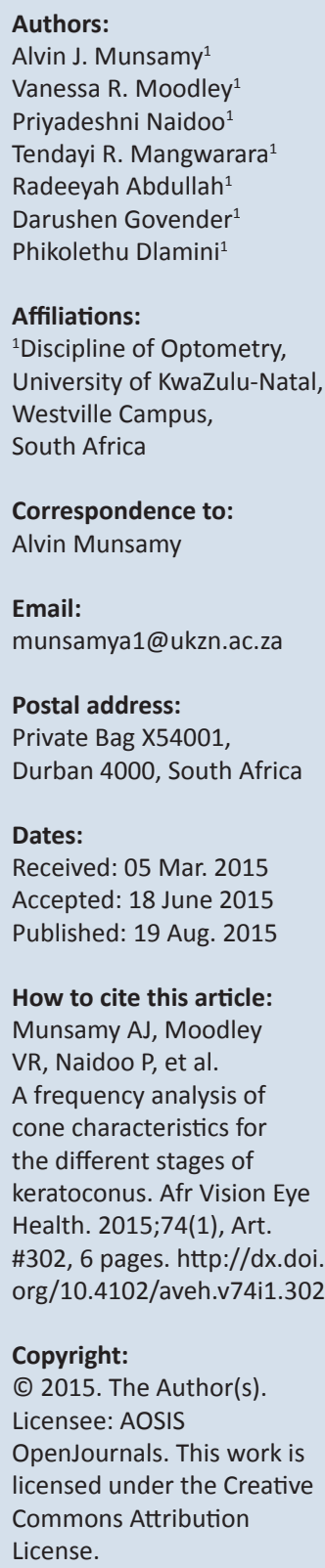

How to cite this article: Munsamy AJ, Moodley VR, Naidoo P, et al. A frequency analysis of cone characteristics for the different stages of keratoconus. Afr Vision Eye Health. 2015;74(1), Art. \#302, 6 pages. http://dx.doi. org/10.4102/aveh.v74i1.302

Copyright:

(C) 2015. The Author(s).

Licensee: AOSIS

OpenJournals. This work is licensed under the Creative Commons Attribution License.

\section{Read online:}

Background: The stages of keratoconus can be classified according to the degree of corneal conicity as either early or advanced, or morphologically by the shape of the cone. Knowledge of the different cone characteristics for the different stages of keratoconus may assist practitioners in diagnosing and managing these patients.

Aim: To describe the cone characteristics for the different stages of keratoconus.

Methods: In this retrospective study, a sample of 190 eyes from 106 cases of previously diagnosed keratoconic patients was analysed. The stage of keratoconus and cone characteristics, namely: cone location, cone decentration, morphology, and topographical patterns were analysed using an Oculus 3M corneal topographer.

Results: Our study revealed that for all stages of keratoconus centrally located cones were the most frequent, with corneal apical decentration between $0 \mathrm{~mm}$ and $2 \mathrm{~mm}$. Frequency analysis revealed that nipple cones were most frequent in all stages. Topographical pattern frequencies revealed a wide distribution amongst the different corneal patterns in all stages of keratoconus.

Conclusion: Cone analysis should be of consideration to the contact lens practitioner when deciding on management of the condition. It additionally serves to compliment the clinical signs such as nerve visibility, Fleischer's ring, Vogt's striae and corneal scarring.

\section{Introduction}

Keratoconus belongs to a group of conditions that cause progressive corneal thinning and distortion. ${ }^{1}$ The most common primary type of corneal ectasia is keratoconus, followed by other differential conditions such as pellucid marginal degeneration and Terrien's degeneration. ${ }^{2}$

Keratoconus is an ocular condition characterized by the progressive non-inflammatory thinning and protrusion of the cornea, ${ }^{2}$ accompanied by the decentration of the resulting cone, corneal distortion, increased irregular astigmatism and myopia. The aetiology and pathogenesis of keratoconus remains inconclusive, ${ }^{3,4}$ with multiple possible causative factors including genetic disorders, hormonal changes, atopic conditions ${ }^{5}$ and, most commonly, biomechanical factors such as eye rubbing. ${ }^{4,6}$

Histopathological features consist of thinning of the corneal stroma, iron deposition in the epithelial basement membrane, breaks in the Bowman's layer, compaction of stromal collagen fibres and folds in Descemet's membrane. ${ }^{7}$ It has been hypothesized that biomechanical factors lead to other histopathological features, such as keratocyte apoptosis, which ultimately causes changes in the cornea and results in a decrease in the total number of keratocytes, the release of degradative enzymes, and the loss of corneal stroma over a period of time. ${ }^{8}$

There are published population-based studies indicating the prevalence of keratoconus in Africa, whilst globally this figure varies widely, depending on the geographic location, diagnostic criteria used and the cohort of patients selected..$^{2,9}$ A review article ${ }^{10}$ in 2012 revealed only two population-based studies by Hofstetter ${ }^{11}$ in the USA and by Jonas ${ }^{12}$ in India. According to this review, Hofstetter reported ${ }^{11}$ a prevalence of 600 per 100 000, and Jonas reported 2300 per 100000. However, it is worth highlighting that genetic and environmental factors influence the differences in frequencies of keratoconus in various populations. ${ }^{2}$

Keratoconus may be classified according to the central corneal steepening as well as the cone morphological and topographical patterns using corneal topography maps. Keratoconus has been classified according to its keratometric readings and/or signs, the distorted mires and size of its cone. ${ }^{1,2}$ The cone characteristics observed during the various stages of keratoconus 
can be categorised as either topographical or morphological. Topographical characteristics include cone location, apex decentration and topographical corneal pattern. The location of the cone may be either central (located within the central $3 \mathrm{~mm}$ zone) or paracentral (located beyond the central $3 \mathrm{~mm}$ zone). ${ }^{5}$ The paracentral location could be further classified as: superior, inferior, nasal, or temporal to the central $3 \mathrm{~mm}$. Studies ${ }^{13,14,15}$ revealed that the keratoconic cone can be found in any location on the cornea, with the manner and motion in which force is applied to the cornea during eye rubbing leading to its decentration. ${ }^{8,16}$

Various corneal topographical patterns have been reported, namely: round, oval, superior steepening, inferior steepening, irregular, symmetric bow tie, symmetric bow tie with skewed radial axes (SB/SRAX), asymmetric bow tie with inferior steepening (AB/IS), asymmetric bow tie with superior steepening and asymmetric bow tie with skewed radial axes (AB/SRAX). ${ }^{8,17}$ Distinguishing the topographical pattern allows the progression of incipient cases of keratoconus to be detected early and differentiated from common misdiagnosed cases of pellucid marginal degeneration and Terrien's marginal degeneration. ${ }^{8,17}$ Topographical patterns are particularly useful in incipient stages for unilateral cases in the undiagnosed eye. ${ }^{8,17}$ Ertan et al. ${ }^{18}$ reported on the frequency of the following corneal topographical patterns in three different age groups. The bowtie in the younger than 21 years old, inferior global cone in those 21-40 years old, and inferior temporal global cones in those older than 40 years old. ${ }^{18}$ Ameerh et al. ${ }^{19}$ reported, independent of staging of keratoconus, global and bowtie cone patterns to be the most common on 210 bilateral keratoconics in a clinic-based population in Jordan.

Morphological characteristics of the keratoconic cone involve the apex and the point of maximal protrusion, and are used to determine the amount of corneal conicity and the degree of keratoconus using corneal apex power. ${ }^{20}$ The cone type is divided into three categories: nipple, oval and globus cones. A nipple cone is a small, near-central cone of $5 \mathrm{~mm}$ or less in diameter, resembling a circular shape in the paracentral region of the cornea, inferior nasally. ${ }^{8,21}$ The rest of the cornea around the cone is normal or flat in comparison. Oval cones have corneal displacement of the apex below the midline, resulting in a cone located mid-peripherally, with a diameter greater than $5 \mathrm{~mm}$, usually in the inferior temporal location. ${ }^{8,21}$ The superior area of the cornea is normal or flatter in comparison to the cone. ${ }^{8}$ The globus cone has a diameter greater or equal to $75 \%$ of the diameter of the cornea. ${ }^{8,21}$

The Collaborative Longitudinal Evaluation of Keratoconus (CLEK) study ${ }^{15}$ was used to stage the progression of keratoconus. Alternatively, this serves to grade to severity of the keratoconus. The study used a multi-centre, prospective, observational design to describe the course of keratoconus on 1209 subjects over a 3 year period. The study produced a table (see Table 1) to stage or grade the keratoconus using the steepest keratometric reading.
The use of corneal topography alone cannot lead to a direct diagnosis of keratoconus as it must be corroborated with clinical signs, symptoms and/or corneal thickness scans. These diagnostic tools help to distinguish and diagnose keratoconus from cases of normal irregular astigmatism and corneal dystrophies. ${ }^{1,2}$ The diagnosis of keratoconus is based on numerous signs and symptoms presenting at various stages according to the severity of the condition. This information will aid in the sequential treatment, ranging from spectacles, contact lenses to various corneal surgeries.

The objectives of this study were to analyse corneal topography maps in order to determine the stage of keratoconus, locate the cone position, analyse the amount of apical decentration from the centre of the cornea, and to ultimately establish if these different cone characteristics were related to different stages of keratoconus.

\section{Methodology}

Patient contact lens record files at the University of KwaZulu-Natal's Eye Clinic, South Africa, underwent a retrospective analysis. This analysis was conducted between the years 2010 to 2014 subsequent to ethical clearance from the School of Health Science Research and Ethics Committee (UKZN). Patients with a diagnosis of keratoconus and good quality corneal topography maps on the Oculus 3M corneal topographer were included in the study sample. This resulted in 106 cases being identified from which a sample of 190 eyes were admitted for analysis.

\section{Materials and methods}

Corneal topography maps of the subject's most recent visit were analysed in order to classify the stage of keratoconus and to locate the apex and position of the cone. These two characteristics were analysed with respect to the right or left eyes. However, no analysis was obtained correlating the location to eye laterality. The sample comprised of either one or both eyes of the same or different subjects depending on the clinical diagnosis of keratoconus. The maps were also used to determine the morphological and topographical characteristics of the cone. The placido topographer was based on the reflection system, in which a placido disc image was projected on the cornea and a camera captured the reflected image. The corneal topographer used in this study was the Oculus 3M.

\section{Staging of keratoconus}

The staging of keratoconus refers to the grading of the severity of the condition. The CLEK grading system (Table 1) is one of many ways used to classify the stages of keratoconus, with numerous studies ${ }^{15,22,23}$ having used this classification. The K-readings for our study were obtained from the topographer (from axial maps) and grouped into mild, moderate or advanced.

\section{Cone apex decentration}

The cone apex is a point location of the steepest point of the cone. Cone apical decentration refers to the distance 
TABLE 1: Collaborative Longitudinal Evaluation of Keratoconus Study classification of keratoconus using the steepest K-reading.

\begin{tabular}{lc}
\hline Staging of Keratoconus & K-Readings (using the steepest K) \\
\hline Mild & $\leq 45.00 \mathrm{D}$ \\
Moderate & $45.00 \mathrm{D}-52.00 \mathrm{D}$ \\
Advanced & $\geq 52.00 \mathrm{D}$ \\
\hline Source: Zadnik K, Barr J, Edrington T, et al. Baseline findings in the collaborative longitudinal
\end{tabular}

Source: Zadnik K, Barr J, Edrington T, et al. Baseline findings in the collaborative longitudin evaluation of keratoconus (CLEK) study. Invest Ophthalmol Vis Sci. 1998;39:2537-2546.

between the centre of the cornea and the apex of the cone, independent of directions such as superior, inferior, nasal, and temporal. The apical decentration was obtained between the linear distance from the centre of the cornea and the apex of the cone on a tangential map. Each map was separately analysed by three researchers to ensure accuracy of the results.

\section{Cone location}

The cone location accounts for the entire cone and was not limited to the cone apex (or tip). Fourier and axial topography maps for each keratoconic eye were analysed to identify the cone location. Fourier maps show the location of the cone in degrees and the decentration of the steepest part of the cone in millimetres from the centre of the cornea. Axial topography maps show the steepest region of the cone, and these were used simultaneously with the Fourier maps in order to locate the apex of the cone. Fourier maps were found to be superior in determining the spherical and astigmatic changes with regards to corneal images. ${ }^{24}$ Zernike maps of the cornea were not considered because of their unreliability in pathological corneas. ${ }^{25,26,27}$ The cone locations were grouped into five categories, namely central, inferior, superior, nasal, and temporal for each eye and classified according to the degree scale (Table 2).

According to Mandell ${ }^{28}$, the cornea is comprised of a central spherical zone called the corneal cap, which was used as a marker to locate the central cones. Thus, cone location was classified as central if the steepest meridian was positioned within the central $3 \mathrm{~mm}$ and paracentral if the steepest meridian was located beyond the central $3 \mathrm{~mm}$ zone. The paracentral cones were further classified as shown in Table 2.

\section{Morphological characteristics}

The morphological patterns were classified into three categories: nipple, oval and globus. ${ }^{17,23}$ A tangential map of the topographer was selected to best distinguish between these three cones. ${ }^{17}$ The cones were identified and then sized in millimetres using the two predominant types of

TABLE 2: Location of the cone (not the corneal apex) for right and left eye using the degree scale.

\begin{tabular}{|c|c|c|}
\hline Cone position & Degrees for right eye $\left({ }^{\circ}\right)$ & Degrees for left eye $\left({ }^{\circ}\right)$ \\
\hline Central & $\begin{array}{l}\text { Central } 3 \mathrm{~mm} \text { of the } \\
\text { cornea }\end{array}$ & $\begin{array}{l}\text { Central } 3 \mathrm{~mm} \text { of the } \\
\text { cornea }\end{array}$ \\
\hline Superior (to the central $3 \mathrm{~mm}$ ) & $45-135$ or SN to ST & $45-135$ or ST to SN \\
\hline Inferior (to the central $3 \mathrm{~mm}$ ) & $225-315$ or IN to IT & $225-315$ or IN to IT \\
\hline Nasal (to the central $3 \mathrm{~mm}$ ) & $316-44$ or IN to SN & $136-224$ or SN to IN \\
\hline Temporal (to the central $3 \mathrm{~mm}$ ) & $136-224$ or ST to IT & $316-44$ or IT to ST \\
\hline
\end{tabular}

colours indicating corneal steepening on the tangential map. Using these two reference points, markings were made to analyse the size of the cone. Three researchers evaluated each map and measured the size independently to categorise it as either nipple $(<5 \mathrm{~mm})$; oval $(>5 \mathrm{~mm})$ and globus $(>75 \%$ of the cornea) cones.

\section{Topographical patterns}

The topographical patterns of the cones were visually analysed using the tangential map of the Oculus topographer. The patterns were qualitatively assessed by observing the steepest portion of the cornea, that is the two 'warmest' colours. The 'best-fit' pattern from 10 templates ${ }^{29}$ was then agreed upon by the three researchers who independently analysed the topographic patterns. Ten different topographical patterns were based on a template by Rabinowitz. The patterns include: round, oval, superior steepening, inferior steepening, irregular, symmetric bowtie with skewed radial axis, asymmetric bowtie with inferior steepening, asymmetric bowtie with superior steepening, asymmetric bowtie with skewed radial axis and symmetric bowtie.

\section{Data analysis}

The Statistical Package for Social Science (version 21) was used to perform the statistical analysis. Frequency distribution histograms were used to describe the targeted variables of cone location, cone apex decentration, cone morphological patterns and cone topographical patterns with the stages of keratoconus.

\section{Results}

The results of this retrospective study of 106 patients (190 eyes) who were clinically diagnosed with keratoconus are presented with respect to the staging of keratoconus and the frequency of cone location, cone apex decentration, cone morphology and cone topographical patterns, respectively.

The average patient age was $25.17 \pm 11.42$ years, ranging from 10 to 60 years old. Gender and ethnicity were not indicated in all patient files thereby excluding their use in analysis.

Regarding the staging (see Table 1), the sample distribution revealed 10 eyes for Stage 1 (mild), 61 eyes for Stage 2 (moderate) and 119 eyes for Stage 3 (advanced) keratoconus. Frequency analysis was used to report the characteristics of the cone for the various stages of keratoconus.

As shown in Figure 1, 66\% of cones were central in Stage 1 (mild keratoconus). In Stage 2 (moderate keratoconus), 50\% were central, and in Stage 3 (advanced keratoconus), 65\% were central.

As shown in Figure 2, 44\% of the cones in Stage 1 (mild keratoconus) were decentered between $0 \mathrm{~mm}-1 \mathrm{~mm}$, $52 \%$ of the Stage 2 (moderate keratoconus) cones were decentered between $0 \mathrm{~mm}-1 \mathrm{~mm}$, and in Stage 3 (advanced 


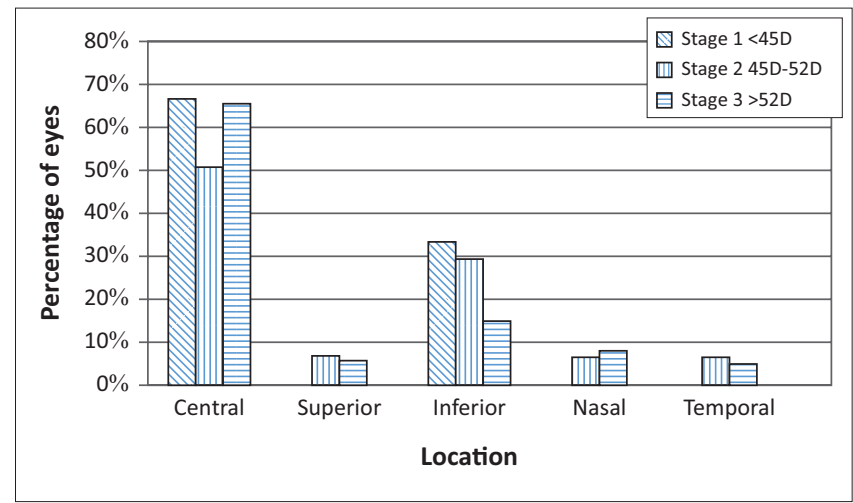

FIGURE 1: The frequency distribution of the cone locations for the various stages of keratoconus.

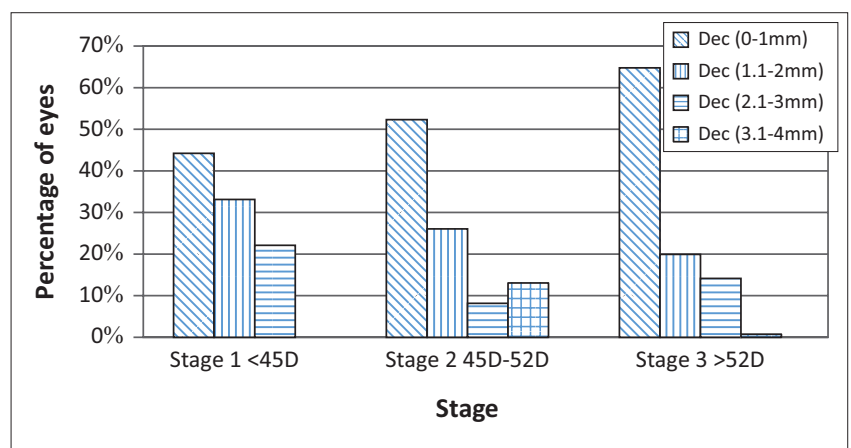

FIGURE 2: The frequency distribution of cone apical decentration and the Stage of keratoconus.

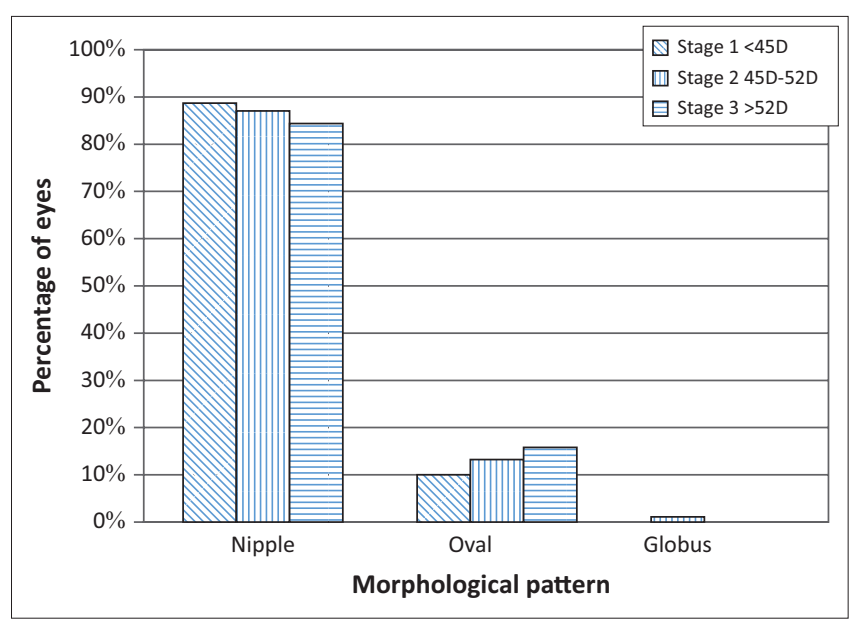

FIGURE 3: The frequency distribution of cone morphological patterns for the Stage of keratoconus.

keratoconus), $65 \%$ of the cones had an apical decentration between $0 \mathrm{~mm}-1 \mathrm{~mm}$.

As shown in Figure 3, the most frequent cone morphology in Stage $1(88 \%), 2(88 \%)$ and $3(84 \%)$ were nipple-shaped cones.

As shown in Figure 4, for Stage 1 (mild keratoconus), the most frequent patterns were round $(36 \%)$ and symmetrical bowtie (36\%). In Stage 2 (moderate keratoconus), the round pattern was most frequent (32\%), and in Stage 3 (advanced keratoconus) the round $(22 \%)$ and irregular (30\%) patterns were most frequent.

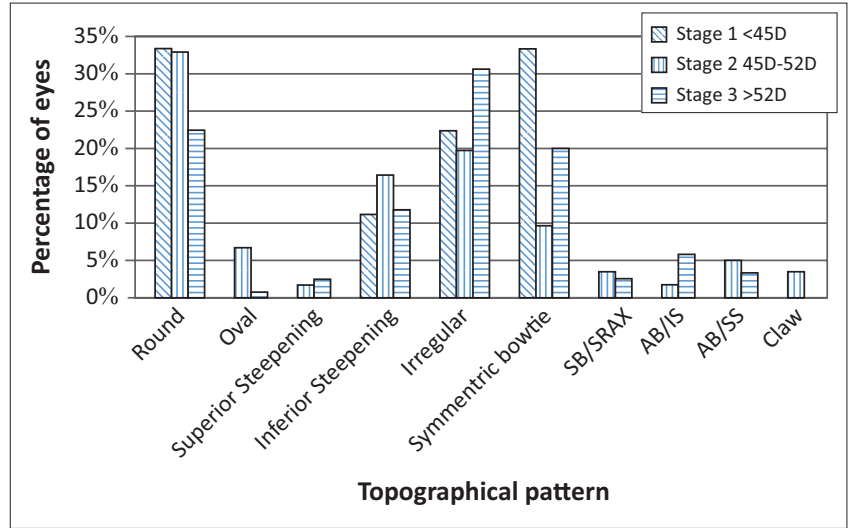

FIGURE 4: The frequency distribution of cone topographical patterns versus stage of keratoconus.

\section{Discussion}

The majority of participants $(76 \%)$ had bilateral keratoconus, suggesting a lower frequency of unilateral keratoconus. Studies in China and the United Kingdom have reported lower incidence of unilateral keratoconus. ${ }^{30,31,32}$ The reporting of cone characteristics using frequency analysis appeared in numerous other studies, ${ }^{13,19,33}$ and our results revealed that the majority of cones were centrally located for all three stages. Additionally there appears to be an inferior displacement of the cone in approximately a third of cases in the mild and moderate stages of keratoconus. A similar finding with smaller samples and no staging of keratoconus was found in other frequency studies. ${ }^{33,34}$ Auffarth et al..$^{35}$ studied 71 eyes and found a mean cone decentration of $0.91 \mathrm{~mm}$. However, their classification differed to that used in our study, in which cone decentration (not cone apical) within the central $3 \mathrm{~mm}$ of the corneal cap was classified as central. Whilst Baum et al. ${ }^{34}$ found paracentral cones to be most frequent, the methodology may have used different criterion for central referencing.

Identifying a definite morphology for a specific stage of the disease can be a challenge because of the multifactorial etiology of keratoconus. Several diseases, such as rosacea, eczema or allergic conjunctivitis, have different pathophysiological mechanisms that produce the corneal changes seen in keratoconus. This study found that the most prevalent morphology was nipple in all three stages of keratoconus. Corneal rubbing will cause epithelial injury and continuous keratocyte apoptosis, causing a decrease in keratocytes as well as releasing degradative enzymes. ${ }^{8}$ Hence, if the cone begins with a nipple morphology, continuous rubbing will cause degradation of the epithelium, resulting in a larger area of thinning, leading to a change in the corneal morphology. ${ }^{16}$ Our finding is similar to other studies, which found that the most prevalent cone locations were central and paracentral, with nipple morphology being associated with these locations. ${ }^{36,37}$

Regarding an association between the stage of keratoconus and cone topography, the results showed round and symmetrical bow tie patterns to be more frequent in the mild stage. The round, irregular and inferior steepening 
patterns were most frequent in the moderate stage, and the most frequent patterns for the severe stage were irregular, round and symmetrical bow tie. Studies ${ }^{8,16}$ suggest the impracticality of predicting what pattern would be expected, as each keratoconic cornea has periods of progression as well as periods of remission. However, topographical patterns have been shown to be useful in diagnosing incipient stages (forme fruste) and differentially diagnosing other forms of corneal ectasia. ${ }^{17,29}$ An observation from this study is that the round topographical pattern has a definite presence in all stages of keratoconus, hence implying for all stages of keratoconus that the steepest part of a keratoconic cornea is round and off-centre. ${ }^{17}$ Clinically this may be useful to the practitioner attempting to fit corneal or scleral contact lenses.

The study attempted to isolate characteristics for the various stages of keratoconus. However, the results indicate that all three stages appear to have a predominance of central cones with cone apical displacement between $0 \mathrm{~mm}$ and $2 \mathrm{~mm}$. Nipple cone morphology was most common in all three stages, as was round topographical patterns, with symmetrical bow tie patterns additionally presenting in Stage 1, and irregular patterns in Stage 3. The results from the frequency analysis of the sample may be useful clinically to predict the most appropriate method of keratoconus management.

\section{Conclusion}

The use of cone analysis has generally not been considered by practitioners when staging keratoconus. The study highlights cone characteristics associated with the different stages of keratoconus and draws attention to the most frequent cone characteristics of cone location, apical decentration, cone morphological shapes, and cone topographical patterns. The results of this study might be used to guide practitioners on which lens designs may be required more frequently in managing these patients towards obtaining optimal visual acuity. The cone analysis additionally serves as a complement to the more commonly cited clinical signs of nerve visibility, Fleischer's ring, Vogt's striae and corneal scarring. ${ }^{15,33}$ Although the study indicates cone characteristics that are more frequent in a South African sample, a study with a larger sample size that also accounts for gender and ethnicity is recommended.

\section{Acknowledgements Competing interests}

The authors declare that they have no financial or personal relationships which may have inappropriately influenced them in writing this article.

\section{Authors' contributions}

A.J.M. (University of KwaZulu-Natal) and V.R.M. (University of KwaZulu-Natal) are academic staff at the University of KwaZulu-Natal. P.N. (University of KwaZulu-Natal); T.R.M. (University of KwaZulu-Natal); R.A. (University of KwaZuluNatal); D.G. (University of KwaZulu-Natal); P.D. (University of KwaZulu-Natal) were undergraduate students at the
University of KwaZulu-Natal. All of the above-mentioned researchers were involved throughout the study.

\section{References}

1. Uçakhan OO, Ozkan M, Kanpolat A. Corneal thickness measurements in normal and keratoconic eyes: Pentacam comprehensive eye scanner versus noncontact specular microscopy and ultrasound pachymetry. J Cataract Refract Surg. 2006;32:970-977. http://dx.doi.org/10.1016/j.jcrs.2006.02.037

2. Barbara A. Textbook on keratoconus: New insights. New Delhi: JP Medical Publishers (P) Ltd, 2011; p. 3-49.

3. Kemp EG, Lewis EJ. Immunoglobulin patterns in keratoconus with particular reference to total and specific IgE levels. Br J Ophthalmol. 1982;66:717-720. http://dx.doi.org/10.1136/bjo.66.11.717

4. Karseras AG, Ruben M. Aetiology of keratoconus. Br J Ophthalmol. 1976;60:522525. http://dx.doi.org/10.1136/bjo.60.7.522

5. Rose P. A systematic approach to fitting keratoconus lenses. Contact Lens Society of America; First Quarter, 2005.

6. Copeman PW. Eczema and keratoconus. Br Med J. 1965;2:977-979. http://dx.doi. org/10.1136/bmj.2.5468.977

7. Fernandes BF, Logan P, Zajdenweber ME, Santos LN, Cheema DP, Burnier MN Jr. Histopathological study of 49 cases of keratoconus. Pathology. 2008;40:623-626. http://dx.doi.org/10.1080/00313020802320648

8. Kim WJL, Rabinowitz YS, Meisler DM, Wilson SE. Keratocyte apoptosis associated with keratoconus. Exp Eye Res. 1999;69:475-481. http://dx.doi.org/10.1006/ exer.1999.0719

9. Gokhale NS. Epidemiology of keratoconus. Indian J Ophthalmol. 2013;61:382383. $h$ ttp://dx.doi.org/10.4103/0301-4738.116054

10. Gordon-Shaag A, Millodot M, Shneor E. The epidemiology and etiology of keratoconus. Int J Keratoco Ectatic Corneal Dis. 2012;1:7-15. http://dx.doi. org/10.5005/jp-journals-10025-1002

11. Hofstetter HW. A keratoscopic survey of 13395 eyes. Am J Optom Arch Am Acad Optom. 1959;36:3-11. http://dx.doi.org/10.1097/00006324-195901000-00002

12. Jonas JB, Nangia V, Martin A, et al. Prevalence and associations of keratoconus in rural Maharashtra in central India: The Central India Eye and Medical Study. Am J Ophthalmol. 2009;148:760-775. http://dx.doi.org/10.1016/j.ajo.2009.06.024

13. Nejabat M, Khalili M, Dehghani C. Cone location and correction of keratoconus with rigid gas-permeable contact lenses. Cont Lens Anterior Eye. 2012;35:17-21. http://dx.doi.org/10.1016/j.clae.2011.08.007

14. McMahon TT, Szczotka-Flynn L, Barr JT, Anderson RJ, Slaughter ME, Lass $\mathrm{JH}$. A new method of grading the severity of keratoconus: The keratoconus severity score (KSS). Cornea. 2006;25:794-800. http://dx.doi.org/10.1097/01. ico.0000226359.26678.d1

15. Zadnik K, Barr J, Edrington T, et al. Baseline findings in the collaborative Iongitudinal evaluation of keratoconus (CLEK) study. Invest Ophthalmol Vis Sci. 1998;39:2537-2546.

16. Sherwin T, Brookes NH. Morphological changes in keratoconus: Pathology or pathogenesis. Clin Experiment Ophthalmol. 2004;32:211-217. http://dx.doi. org/10.1111/j.1442-9071.2004.00805.x

17. Sinjab M. Quick guide to the management of keratoconus. Berlin, Heidelberg: Springer-Verlag; 2012. http://dx.doi.org/10.1007/978-3-642-21840-8

18. Ertan AL, Kamburoglu G, Colin J. Location of steepest corneal area of cone in keratoconus stratified by age using Pentacam. J Refract Surg. 2009;25:1012-1016. http://dx.doi.org/10.3928/1081597X-20091016-07

19. Abu Ameerh MA, Hamad Gl, Albdour MD. Topographic characteristics of keratoconus among a sample of Jordanian patients. Int J Ophthalmol. 2014;7:714-719.

20. Armitage JA, Bruce AS, Phillips AJ, Lindsay RG. Morphological variants in keratoconus: Anatomical observation or aetiologically significant? Aust N Z J Ophthalmol. 1998;S1:S68-S70. http://dx.doi.org/10.1111/j.1442-9071.1998.tb01378.x

21. Corbett MC. Corneal topography: Basic principles and applications to refractive surgery. Optometry Today. 2000;899-905.

22. Saini J, Saroha V, Singh P, Sukhija J, Jain A. Keratoconus in Asian eyes at a tertiary eye care facility. Clin Experiment Ophthalmol. 2004;87:97-101. http://dx.doi. org/10.1111/j.1444-0938.2004.tb03155.x

23. Gupta D. Keratoconus: A clinical update. Optometry Today. 2005:34-37.

24. Hjortdal J, Erdmann L, Bek T. Fourier analysis of video-keratographic data. A tool for separation of spherical, regular astigmatic and irregular astigmatic corneal power components. Ophthalmic Physiol Opt. 1995;15:171-185.

25. Carvalho LA. Accuracy of Zernike polynomials in characterizing optical aberrations and the corneal surface of the eye. Invest Ophthalmol Vis Sci. 2005;46:19151926. http://dx.doi.org/10.1167/iovs.04-1222

26. Smolek MK, Klyce SD. Zernike polynomial fitting fails to represent all visually significant corneal aberrations. Invest Ophthalmol Vis Sci. 2003;44:4676-4681. http://dx.doi.org/10.1167/iovs.03-0190

27. Klyce SD, Karon MD, Smolek MK. Advantages and disadvantages of the Zernike expansion for representing wave aberration of the normal and aberrated eye. $J$ Refract Surg. 2004;20:S537-S541.

28. Mandell RB. Contact lens practice. 4th ed. Springfield, IL: Thomas; 1974; p. 107-109. 
29. Rabinowitz Y. Diagnosing keratoconus and patients at risk. Cataract Refract Surg Today. 2007;85-87.

30. Wei R, Zhao S, Lim L, Tan D. Incidence and characteristics of unilateral keratoconus classified on corneal topography. J Refract Surg. 2011;27:745-751. http://dx.doi.org/10.3928/1081597X-20110426-01

31. Khor WB, Wei RH, Lim L, Chan C, Tan D. Keratoconus in Asians: Demographics, clinical characteristics and visual function in a hospital based population. Clin Experiment Ophthalmol. 2011;39:299-307. http://dx.doi.org/10.1111/ j.1442-9071.2010.02458.x

32. Burns DM, Johnston FM, Frazer DG, Patterson C, Jackson AJ. Keratoconus: An analysis of corneal asymmetry. Br J Ophthalmol. 2004;88:1252-1255. http:// dx.doi.org/10.1136/bjo.2003.033670
33. Gillan WDH. Keratoconus: A clinical exposé. S Afr Optom. 2013;72:41-45.

34. Baum J. On the location of the cone and the etiology of keratoconus. Cornea. 1995;14:142-143. http://dx.doi.org/10.1097/00003226-19950300000004

35. Auffarth GU, Wang L, Völcker HE. Keratoconus evaluation using the Orbscan topography system. J Cataract Refract Surg. 2000;26:222-228. http://dx.doi. org/10.1016/S0886-3350(99)00355-7

36. Wilson SE, David TC, Lin S, Klyce D. Corneal topography of keratoconus. Cornea. 1991;10:2-8. http://dx.doi.org/10.1097/00003226-199101000-00002

37. Rabinowitz YS. Keratoconus. Surv Ophthalmol. 1998;42:297-319. http://dx.doi. org/10.1016/S0039-6257(97)00119-7 